\title{
Distribuição espacial da cigarrinha Empoasca kraemeri Ross \& Moore (Hemiptera: Cicadelidae) no feijão-de-corda e cálculo do número de amostras
}

\author{
Spatial distribution of leafhopper Empoasca kraemeri Ross \& Moore \\ (Hemiptera: Cicadelidae) in cowpea and calculation of number of samples
}

\author{
Valéria Silva ${ }^{1 *}$, Gleidson Vieira Marques ${ }^{2}$, Jefté Ferreira da Silva ${ }^{3}$, Ervino Bleicher ${ }^{1}$ \\ | | | | | | | | | | | | | | | | | | | | | | | | | | | | | | | | | | | | | | | | | | | | | | | | | | | | | | | | | | | | | | | | | | | | | | | | | | | | | | | | | | | | | | | | | | | | | | | | | | | | | | | | | | | | | | | | | | | | | | | | | | | | | | | | | | | | | | | | | | | | | | | | | | | | | | | | | | | | | | | | | | | | | | | | | | | | | | | | | | | | | | | | | | | | | | | | |
}

RESUMO: Objetivou-se estudar a dispersão espacial da cigarrinha Empoasca kraemeri Ross \& Moore (Hemiptera: Cicadelidae) na cultura de feijão-de-corda Vigna unguiculata (L.) Walp. e estabelecer o número de amostras necessárias para a estimativa da populaçáo da praga para o uso em programas de manejo integrado de pragas. Foram cultivados dois campos experimentais na Universidade Federal do Ceará, em Fortaleza. O primeiro campo com área de $216 \mathrm{~m}^{2}$ composta de 15 parcelas. O segundo campo com área de $576 \mathrm{~m}^{2}$ dividida em 25 parcelas. O cultivar utilizado foi Vita 7 com plantas espaçadas em 0,25 x 0,8 m. Foram realizadas três coletas de dados no Campo I e quatro no Campo II, sendo avaliadas dez plantas por parcela. Foi contado o número de adultos e ninfas de cigarrinha presentes em toda a planta. Os resultados obtidos nos índices de agregação utilizados indicam que a dispersão da E. kraemeri no campo é do tipo agregada, o que foi confirmado pelo ajuste dos dados à distribuiçáo de frequência binomial negativa. Para aplicação em programas de manejo integrado de pragas, 30 é o número de amostras estatisticamente adequado para a estimativa da populaçáo de E. kraemeri em campos de V. unguiculata.

PALAVRAS-CHAVE: Vigna unguiculata; amostragem; distribuição horizontal; manejo integrado de pragas.

\begin{abstract}
The objective was to study the spatial dispersion of the leafhopper Empoasca kraemeri Ross \& Moore (Hemiptera: Cicadelidae) on culture of cowpea Vigna unguiculata (L.) Walp. and to establish the number of sample necessary to estimate the population of the pest for use in integrated pest management programs. Two experimental fields were cultivated at the Federal University of Ceará, in Fortaleza, Brazil. The area of the Field I was $216 \mathrm{~m}^{2}$, composed of 15 plots. The area of the Field II was $576 \mathrm{~m}^{2}$, divided into 25 plots. The cultivar used was Vita 7 with plants spaced $0.25 \times 0.8 \mathrm{~m}$. We performed three separate collections of data in Field I and four in Field II; in each plot ten plants were assessed. The number of leafhopper nymphs and adults present in the plant was counted. The aggregate indexes used indicate that the dispersion of E. kraemeri in the field is of the aggregate type, which was confirmed by fitting the data to the negative binomial frequency distribution. To be used in integrated pest management programs, 30 is the sample number statistically suitable for the estimation of E. kraemeri population in V. unguiculata fields.
\end{abstract}

KEYWORDS: Vigna unguiculata; sampling; horizontal distribution; integrated pest management. 


\section{INTRODUÇÃO}

Entre as principais pragas do feijão-de-corda, destaca-se a Empoasca kraemeri Ross \& Moore (Hemiptera: Cicadelidae), comumente conhecida por cigarrinha verde do feijoeiro, que provoca danos de ordem física como consequência da penetração do estilete no floema da planta, ocasionando a desorganização e granulação das células e obstrução dos vasos condutores de seiva. Além disso, ocorre também a injeção de substâncias toxicogênicas durante a alimentação (PEREIRA et al., 1993). Segundo esses autores, as altas infestaçóes da praga levam ao "enfezamento" ou redução do porte das plantas, que passam a mostrar as bordas dos folíolos viradas para baixo. Em casos mais severos ocorre o amarelecimento das margens dos folíolos e posterior secamento dessas estruturas.

Em relação ao controle, vem-se reduzindo o uso de inseticidas e evitando-se a aplicação sistemática de produtos químicos em culturas de importância agrícola que tomava como base o uso de calendários preestabelecidos fazendo com que, muitas vezes, a aplicação fosse feita sem mesmo a praga estar presente (GALlo et al., 2002).

$\mathrm{O}$ manejo integrado de pragas (MIP) representa um avanço significativo como sistema racional de controle de pragas, pois tem como principal objetivo a utilização mínima de agroquímicos (Herrera, 2010). No contexto do MIP, a tomada de decisão é um aspecto chave e básico para se decidir sobre a necessidade ou não de alguma ação de controle com base na densidade da praga e, de forma ideal, na densidade dos inimigos naturais também. Assim, para que o MIP seja empregado satisfatoriamente é fundamental estimar a densidade da praga, e para isso é necessário o conhecimento de uma forma de amostragem rápida e eficiente das pragas e seus inimigos naturais (Fernandes et al., 2003), incluindo o momento apropriado de utilização de práticas de controle químico quando as populaçóes alcançam o nível de limiar econômico.

A determinação de um número adequado de amostras a serem tomadas em um programa de manejo é normalmente baseado em uma relação entre a precisão e o custo da amostragem. Para se obter esse valor, um número considerável de pesquisas pode ser requerido para determinar o número de amostras necessárias que seja economicamente praticável (Pedigo; Rice, 2009).

Um confiável programa de amostragem inclui a definição do momento adequado para a avaliação; unidade amostral; definição do padrão de dispersão espacial; bem como o número de amostras (Pedigo; Buntin, 1994; Southwood; Henderson, 2000). Assim, o conhecimento da distribuição espacial de populaçóes de insetos é fundamental para o desenvolvimento de planos de amostragens (Giles et al., 2000), com a finalidade de aplicação em MIP.

A determinação da distribuição espacial pode ser obtida através das distribuiçóes de frequência e do cálculo de índices de dispersão. Estes últimos, embora não descrevam matematicamente a distribuição da população estudada (ELliotT et al., 1990), fornecem uma ideia bastante aproximada da tendência de dispersão quando diferentes índices fornecem resultados similares. Dentre os índices de dispersão mais empregados tem-se o de razão variância/média, índice de Morisita, coeficiente de Green e expoente $\mathrm{k}$ da binomial negativa (RAhman et al., 2010; SEdARATian et al., 2010; Cesconetto et al., 2005).

A distribuição espacial de uma população pode ser de três tipos: agregada (ou contagiosa), aleatória (ou ao acaso) ou uniforme (ou regular). Esses tipos normalmente seguem um modelo matemático, feito com base na relação entre a variância e a média dos dados, que descreve uma distribuição de probabilidade (Maruyama et al., 2006). Tais distribuiçôes de probabilidades são: binomial negativa (agregada), Poisson (aleatória) e binomial positiva (uniforme).

Assim, objetivou-se com este trabalho estudar a distribuição espacial da E. kraemeri e determinar um número de amostras aplicáveis ao MIP em cultivo de $V$. unguiculata.

\section{MATERIAL E MÉTODOS}

Para a realização do estudo foram instaladas duas áreas experimentais, ambas no Campus do Pici, na Universidade Federal do Ceará, em Fortaleza ( $3^{\circ} 40^{\prime}$ S $38^{\circ} 34^{\prime}$ O, altitude $12 \mathrm{~m}$ acima do nível do mar). O período de condução dos experimentos foi de 11 de abril a 3 de junho de 2011, sendo utilizado o cultivar Vita 7 de ciclo precoce, deixando-se apenas uma planta por cova. O espaçamento utilizado foi de $0,25 \times 0,8 \mathrm{~m}$, sendo a área total do primeiro experimento (Campo I) de $216 \mathrm{~m}^{2}$ com 15 parcelas de dimensão 4 x 3,6 m; e a área total do segundo experimento (Campo II) de $576 \mathrm{~m}^{2}$ contendo 25 parcelas separadas por fitas de nylon em ambos os experimentos. Foi realizada uma adubação aos 14 dias após a semeadura, que se constituiu da aplicação de $10 \mathrm{~kg} \cdot \mathrm{ha}^{-1}$ de nitrogênio, $10 \mathrm{~kg} \cdot \mathrm{ha}^{-1}$ de fósforo e $10 \mathrm{~kg} \cdot \mathrm{ha}^{-1}$ de potássio, provenientes dos adubos ureia, super fosfato triplo e cloreto de potássio, respectivamente.

Para o estudo da distribuiçáo espacial, os dados foram obtidos mediante contagem do número de adultos e ninfas de cigarrinhas presentes em toda a planta, sendo que, em cada parcela, foram avaliadas dez plantas em uma linha central.

Foram feitas avaliaçôes semanais, sendo utilizadas para o Campo I as avaliaçóes dos dias 20/05/2011, 27/05/2011 e 03/06/2011 e no Campo II as avaliaçóes dos dias 13/05/2011, 20/05/2011, 27/05/2011 e 03/06/2011.

Os índices de dispersão utilizados para verificar a distribuição da cigarrinha no feijoeiro, foram:

- Razão variância/media (I), índice calculado por: 
$I=\frac{s^{2}}{m}$

onde:

$s^{2}$ : variância amostral;

$m$ : média amostral.

- Significância da diferença da unidade (teste de afastamento da aleatoriedade), testada através da determinação do valor da estatística do qui-quadrado $\left(\chi^{2}\right)$ pela expressão:

$\chi^{2}=I(N-1)$

onde:

I: valor do índice de dispersão I;

$N$ : número total de amostras.

- Teste de afastamento da aleatoriedade, que consiste em rejeitar a aleatoriedade se:

$(N-1)>\chi_{(N-1 g, 1, \alpha)}^{2}$

onde:

g.l.: graus de liberdade.

- Valor "d". Para amostras grandes (n > 31) a aceitação, ou náo, da aleatoriedade vai depender do módulo de "d". O valor " $d$ " foi calculado por:

$|d|=\sqrt{2 \chi^{2}}-\sqrt{2 v-1} \geq Z_{\alpha}$

onde:

$\chi^{2}$ : estatística do teste qui-quadrado para o índice $I$;

$v$ : número de graus de liberdade;

$Z$ : valor da distribuição normal padrão ao nível alfa de probabilidade.

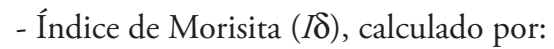

$I_{\delta}=\frac{N\left(\sum_{i=1}^{N} x_{\mathrm{i}}^{2}-\sum_{i=1}^{N} x_{\mathrm{i}}\right)}{\left(\sum_{i=1}^{N} x_{\mathrm{i}}\right)^{2}-\sum_{i=1}^{N} x_{\mathrm{i}}}$

onde:

$N$ : número total de amostras;

$x_{i}$ : número de insetos na $i$-ésima unidade amostral.

- Afastamento da aleatoriedade, que foi testado pela expressão:

$\chi_{\delta}^{2}=I_{\delta}\left(\sum_{i=1}^{N} x_{\mathrm{i}}-1\right)+N-\sum_{i=1}^{N} x_{i}$ onde:

$I_{\delta}$ índice de dispersão de Morisita;

$N$ : número total de amostras;

$x_{i}$ : número de insetos na $i$-ésima unidade amostral.

O valor de $\chi^{2}$ para o índice de Morisita foi comparado com o valor de $\chi_{(N-1,1.1, \alpha)}^{2}$, rejeitando-se a aleatoriedade à distribuição estudada se:

$\chi_{\delta}^{2}>\chi_{(N-1 g .1, \alpha)}^{2}$

onde:

g.l.: graus de liberdade.

- Coeficiente de Green (Cx), que foi dado por:

$C_{x}=\frac{\left(\frac{s^{2}}{m}\right)-1}{\sum_{i=1}^{N} x_{i}-1}$

onde:

$s^{2}$ : variância amostral;

$m$ : média amostral;

$x_{i}$ : número de insetos na $i$-ésima unidade amostral.

O afastamento da aleatoriedade é testado pela expressão:

$C_{x,(1-\alpha)}=\frac{\left(\frac{\chi_{(4-\alpha)}}{(N-1)}\right)-1}{N \times m-1}$

onde:

$x^{2}{ }_{(1-\alpha)}$ : valor do qui-quadrado com N-1 graus de liberdade e um nível $\alpha$ de significância;

$N$ : número total de amostras;

$m$ : média amostral.

- Expoente $\mathrm{k}$ da distribuição binomial negativa, calculado por:

$k=\frac{m^{2}}{s^{2}-m}$

onde:

$s^{2}$ : variância amostral;

$m$ : média amostral.

Para confirmar o tipo de distribuição da cigarrinha no feijão-de-corda foram feitas as distribuiçóes de frequência dos números de indivíduos da praga. Em função de todos os índices de dispersão sugerirem distribuição agregada, optou-se por fazer apenas as distribuiçóes teóricas de frequências de 
Poisson e binomial negativa. A distribuição de Poisson foi calculada por:

$$
\begin{aligned}
& P(0)=e^{-m} \\
& P(x)=\frac{m}{x} \times P(x-1) \quad x=1,2,3 \ldots \alpha
\end{aligned}
$$

onde:

$P(x)$ : probabilidade de encontrar $x$ indivíduos em uma unidade amostral;

$e$ : base do logaritmo neperiano $(2,718282 \ldots)$;

$m$ : média amostral.

A distribuição de probabilidade binomial negativa foi calculada por:

$$
\begin{aligned}
& P(0)=\left(1+\frac{m}{k}\right)^{-k} \\
& P(x)=\frac{k+x-1}{x} \times\left(\frac{m}{m+k}\right) \times P(x-1) \quad x=1,2,3 \ldots, \mathrm{a}
\end{aligned}
$$

onde:

$k$ : expoente $\mathrm{k} \mathrm{da}$ binomial negativa;

$P(x)$ : probabilidade de encontrar $x$ indivíduos em uma unidade amostral;

$m$ : média amostral.

Para se verificar o ajuste dos dados obtidos nas distribuiçóes de Poisson e binomial negativa utilizou-se o teste do qui-quadrado, que consiste em comparar as frequências observadas com as frequências esperadas:

$$
\chi^{2}=\sum_{i=1}^{n_{c}} \frac{\left(F O_{i}-F E_{i}\right)^{2}}{F E_{i}}
$$

onde:

$n_{\mathrm{c}}$ : número de classes da distribuição de frequências;

$F O i$ : frequência observada na $i$-ésima classe;

$F E i$ : frequência esperada na $i$-ésima classe.

Foi fixado, para a realização desse teste, uma frequência esperada mínima igual a 1 . O número de graus de liberdade associado à estatística $\chi^{2}$ foram determinados por:

$G L=N_{c}-N_{p}-1$

onde:

$G L:$ número de graus de liberdade;

$N$ : número de classes da distribuição de frequências;
$N p$ : número de parâmetros estimados na amostra para o calculo da distribuição.

O valor de $\chi^{2}$ foi comparado com o valor de $\chi_{\left(n-1 g, 1, x_{\alpha=0.05)}\right.}^{2}$ rejeitando-se o ajuste à distribuição estudada, se $\chi^{2} \geq \chi_{\left(n-1,1, l_{\alpha}\right)^{\circ}}$.

Para o cálculo do número de amostras foram utilizadas as avaliaçôes dos dias 20/05/2011, 27/05/2011 e 03/06/2011 do Campo I e 20/05/2011, 27/05/2011 e 03/06/2011 do Campo II. Na avaliação do dia 13/05/2011 no Campo II observou-se uma baixa infestação, apresentando muitas amostras com valor zero, não fornecendo uma boa amostra para a aplicação nas fórmulas.

O número de amostras foi calculado com base na avaliação da variação relativa $(V R)$, calculada por:

$V R=\frac{s_{x}}{m} \times 100$

onde:

$V R$ : variação relativa;

$s_{\mathrm{x}}$ : erro padrão da média;

$m$ : média amostral.

O número de amostras também foi estimado utilizando-se a fórmula proposta para quando a distribuição espacial do inseto é agregada (Kogan; Herzog, 1980), sendo:

$n=\left[\frac{1}{x}+\left(\frac{1}{k}\right)^{2}\right] \times \frac{t^{2}}{D^{2}}$

onde:

$k$ : expoente $k$ da binomial negativa;

$x$ : número de indivíduos nas amostras;

$D$ : nível de precisão estabelecido $(V R)$ expressado como decimal;

t: valor do teste t para $P=\alpha$.

Com o objetivo de obter uma quantidade de amostras aplicáveis ao MIP, considerou-se uma precisão de $25 \%$, conforme sugerido por Southwood; Henderson (2000).

Todas essas análises foram realizadas utilizando o programa para computador Microsoft Excel $^{\circledR} 2010$.

\section{RESULTADOS E DISCUSSÃO}

A razão variância/média ( $I$ ) apresentou valores maiores do que a unidade em todas as avaliaçóes, indicando o modelo de distribuição agregada da cigarrinha do feijāo-de-corda no campo. Essa agregação é confirmada pelos valores significativos $(\mathrm{p}<0,01)$ da estatística $|\mathrm{d}|$ diferenciando-o da 
aleatoriedade. $\mathrm{O}$ índice de Morisita $\left(\mathrm{I}_{\delta}\right)$ foi superior à unidade em todas as avaliaçóes, com uma significância de 1\% no afastamento da aleatoriedade, indicando, novamente, o modelo de distribuição agregada. $\mathrm{O}$ índice $\mathrm{k}$ da binomial negativa, em três das sete avaliaçôes, indicou alta agregação do inseto $(0<\mathrm{k} \leq 2)$. Nas outras quatro avaliaçôes o índice indicou agregação $(2<\mathrm{k} \leq 8)$, caracterizando o tipo de distribuição agregada. Os valores do índice de Green $\left(\mathrm{C}_{\mathrm{x}}\right)$ também foram significativos, a $1 \%$ de probabilidade, mostrando a agregação de E. kraemeri (Tabela 1).

Apesar dos índices de agregação ou dispersão não descreverem matematicamente a distribuição da populaçấo estudada (ELLIOTT et al., 1990), fornecem uma ideia bastante aproximada dessa realidade quando diferentes índices fornecem resultados similares (Myers, 1978). No presente trabalho, para os dois campos experimentais, todos os índices utilizados sugerem uma distribuição agregada da E. kraemeri no feijāo-de-corda. Entretanto, a confirmação do tipo de distribuição ocorrerá apenas com o conhecimento das distribuiçóes de frequência dos números de indivíduos da praga estudada (BARBOSA, 1992).

A distribuição da população de E. kraemeri não se ajustou à distribuiçáo de Poisson, demonstrando que os insetos não estâo distribuídos aleatoriamente no campo. Em apenas uma das sete amostragens (no dia 03/06/2011 no Campo I) obteve-se um ajuste para essa distribuiçáo. Nas outras seis amostragens a diferença significativa foi ao nível de $1 \%$ de probabilidade. Por outro lado, observa-se um ajuste para a distribuiçấo binomial negativa. Em todas as amostragens a população de cigarrinha apresentou arranjo agregado de seus indivíduos (Tabela 2).

A avaliaçáo do Campo I no dia 03/06/2011 apresentou bom ajuste para ambas as distribuiçóes. Entretanto, no âmbito da estatística ecológica, o melhor ajuste é representado pela distribuiçấo de frequência que apresenta o menor valor de $\chi^{2}$ calculado (Melo et al., 2006). Nesse caso, a binomial negativa (Tabela 2).

Diante dos resultados obtidos, a tendência de distribuição espacial agregada apontada pelos índices foi comprovada pelo modelo teórico da distribuição de frequência, ajustando-se à binomial negativa.

A distribuição de frequência binomial negativa foi relatada para Empoasca kraemeri na cultura do feijoeiro comum em 31 plantios comerciais de feijão, no município de Coimbra, Minas Gerais, após coletas de 451 amostras

Tabela 1. Média, variância, razão variância/média, índice de Morisita, expoente k da binomial negativa e coeficiente de Green para distribuição espacial Empoasca kraemeri em Vigna unguiculata (L.) Walp. Fortaleza, Ceará, 2011.

\begin{tabular}{|c|c|c|c|c|c|c|c|c|c|c|c|}
\hline \multirow{4}{*}{ ô } & \multirow{2}{*}{$\begin{array}{l}\text { Datas } \\
\text { 20/05/2011 }\end{array}$} & \multirow{2}{*}{$\begin{array}{l}\text { Média } \\
\qquad 2,77\end{array}$} & \multirow{2}{*}{$\begin{array}{l}\text { Variância } \\
\text { 7,12 }\end{array}$} & \multicolumn{2}{|c|}{$\begin{array}{l}\text { Razão variância/ } \\
\text { média } \\
\text { । }\end{array}$} & \multicolumn{2}{|c|}{$\begin{array}{l}\text { Índice de Morista } \\
I_{\delta}\end{array}$} & \multicolumn{2}{|c|}{$\begin{array}{c}\text { Expoente } k \text { da } \\
\text { binomial negativa }\end{array}$} & \multicolumn{2}{|c|}{$\begin{array}{l}\text { Coeficiente de Green } \\
C_{x}\end{array}$} \\
\hline & & & & 2,573 & ** & 1,57 & ** & 1,75842 & AAG & 0,00380 & ** \\
\hline & $27 / 05 / 2011$ & 8,28 & 34,32 & 4,145 & ** & 1,38 & $* *$ & 2,63243 & AG & 0,00253 & $* *$ \\
\hline & 03/06/2011 & 25,59 & 197,46 & 7,715 & $* *$ & 1,26 & ** & 3,81111 & AG & 0,00175 & ** \\
\hline \multirow{4}{*}{ 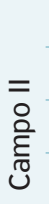 } & $13 / 05 / 2011$ & 0,43 & 1,23 & 2,839 & $* *$ & 5,28 & $* *$ & 0,23496 & AAG & 0,01718 & $* *$ \\
\hline & $20 / 05 / 2011$ & 7,88 & 29,14 & 3,696 & $* *$ & 1,34 & ** & 2,92435 & AG & 0,00137 & ** \\
\hline & 27/05/2011 & 9,79 & 61,90 & 6,324 & $* *$ & 1,54 & ** & 1,83835 & AAG & 0,00218 & $* *$ \\
\hline & 03/06/2011 & 8,51 & 33,64 & 3,952 & $* *$ & 1,35 & $* *$ & 2,88335 & AG & 0,00139 & $* *$ \\
\hline
\end{tabular}

**Teste do qui-quadrado significativo a $1 \%$ de probabilidade; ${ }^{A A G}$ altamente agregado; ${ }^{\mathrm{AG}}$ agregado.

Tabela 2. Teste do qui-quadrado de aderência das frequências observadas e esperadas pelas distribuições de Poisson e binomial negativa para Empoasca kraemeri, em Vigna unguiculata (L.) Walp. Fortaleza, Ceará, 2011.

\begin{tabular}{|c|c|c|c|c|c|}
\hline & \multirow{2}{*}{ Data } & \multicolumn{2}{|c|}{ Poisson } & \multicolumn{2}{|c|}{ Binomial negativa } \\
\hline & & $\chi^{2}$ & Grau de liberdade & $\chi^{2}$ & Grau de liberdade \\
\hline \multirow{3}{*}{$\begin{array}{l}\bar{\circ} \\
\stackrel{\varrho}{E} \\
\text { ठ }\end{array}$} & $20 / 05 / 2011$ & $116,74^{* *}$ & 9 & $19,24^{\text {ns }}$ & 12 \\
\hline & $27 / 05 / 2011$ & $105,02 * *$ & 26 & $10,28^{\text {ns }}$ & 25 \\
\hline & 03/06/2011 & $56,96^{\mathrm{ns}}$ & 82 & $44,91^{\mathrm{ns}}$ & 81 \\
\hline \multirow{4}{*}{$\begin{array}{l}= \\
\bar{\circ} \\
\text { 를 } \\
\text { ठ․ }\end{array}$} & $13 / 05 / 2011$ & $83,71^{* *}$ & 7 & $14,92^{\text {ns }}$ & 6 \\
\hline & $20 / 05 / 2011$ & $118,34^{* *}$ & 26 & $17,28^{\text {ns }}$ & 25 \\
\hline & $27 / 05 / 2011$ & $135,82^{* *}$ & 65 & $29,98^{\text {ns }}$ & 64 \\
\hline & 03/06/2011 & $139,88^{* *}$ & 32 & $31,57^{\text {ns }}$ & 31 \\
\hline
\end{tabular}


por cultivo com batida de duas plantas em bandeja plástica (Moura, 2005) e para Empoasca decipiens (Hemiptera: Cicadellidae) em diferentes espécies de feijāo no Irã durante 2004 e 2005 (NASERI et al., 2009).

Considerando a distribuição espacial do tipo agregada, e aplicando um nível de precisão de $25 \%$, inferimos que o número de amostras, sem risco para o produtor, é de 30 (Tabela 3).

A perda de parte da precisão em favor da rapidez da avaliação é aceita por alguns autores (Southwood, 1966; Southwood; Henderson, 2000), mas estes propuseram outra forma de calcular o número de amostras, mediante o uso da equaçáo da $V R$. Para esses autores, uma $V R$ de até $25 \%$ de precisão poderia ser usada para decisão nos programas de MIP. No entanto, para estudos mais intensivos, como os de pesquisa, onde é exigida precisão maior, a $V R$ deveria situar-se abaixo de $10 \%$.

Como a intenção desta pesquisa é fornecer subsídios ao MIP aplicando a equaçáo da $V R$ até a obtenção de uma precisão menor ou igual a $25 \% \mathrm{em}$ cada data de amostragem, inferimos que o número de amostras de E. kraemeri a serem tomadas é 15 (Tabela 4).

O modelo de distribuição agregado, ao qual a cigarrinha $E$. kraemeri se ajustou melhor, exige um maior número de amostras do que qualquer outro tipo de distribuição espacial na realização de um processo de amostragem (Rodrigues et al., 2010). Entretanto, se for considerado sob o ponto de vista da aplicação desses resultados em uma prática rotineira relativa à avaliação de campos submetidos ao MIP (Southwood; Henderson, 2000), os resultados baseados em Kogan; Herzog (1980) superestimaram os números de amostras necessárias.

Por outro lado, os resultados alcançados mediante o uso da equaçáo de $V R$ nos permite uma indicação segura de um menor número de amostras. Assim, diante desses resultados, pode-se sugerir um número de 15 amostras durante todo o ciclo da cultura, tomando-se como unidade amostral toda a planta. Esse valor é inferior ao sugerido por Moura et al. (2007), de 63 amostras/lavoura de adultos e 118 amostras/ lavoura para amostragem de ninfas de E. kraemeri no feijão comum, pela batida de plantas em bandeja.

Baseando-se apenas na precisão em função da distribuição espacial e ainda sendo conservador, seriam necessárias 30 amostras para quantificar a densidade da cigarrinha pelo método de contagem direta, resultado inferior ao sugerido por Moura et al. (2007), requerendo portanto menor tempo de amostragem. Essa informação é de fundamental importância para o MIP, já que segundo vários autores (Southwood, 1966; Southwood; Henderson, 2000) a rapidez de uma avaliação tem implicaçôes econômicas importantes.

O tempo gasto na amostragem deve ser otimizado de modo que as etapas de coleta, processamento dos dados e tomada de decisão ocorram no mesmo período do dia, pois as atividades requeridas pelo cultivo do feijão, como adubação, controle fitossanitário, irrigação e colheita, demandam grande utilizaçáo de mão de obra, precisando ser adotadas num
Tabela 3. Número de amostras de Empoasca kraemeri, obtidas pela equação proposta por Kogan e Herzog (1980) com um índice de precisão de 25\%. Fortaleza, Ceará, 2011.

\begin{tabular}{|c|c|c|}
\hline & Data & Número de amostras \\
\hline \multirow{3}{*}{$\begin{array}{l}\bar{o} \\
\stackrel{0}{\bar{c}} \\
\text { তु }\end{array}$} & $20 / 05 / 2011$ & 30 \\
\hline & $27 / 05 / 2011$ & 10 \\
\hline & $03 / 06 / 2011$ & 5 \\
\hline \multirow{3}{*}{$\begin{array}{l}\overline{\bar{O}} \\
\stackrel{0}{E} \\
\frac{0}{0} \\
\tilde{U}\end{array}$} & $20 / 05 / 2011$ & 9 \\
\hline & $27 / 05 / 2011$ & 17 \\
\hline & 03/06/2011 & 10 \\
\hline
\end{tabular}

Tabela 4. Número de amostras de Empoasca kraemeri necessárias para uma variância relativa menor ou igual a 25\%. Fortaleza, Ceará, 2011.

\begin{tabular}{|c|c|c|c|}
\hline & Data & $\begin{array}{l}\text { Número de } \\
\text { amostras }\end{array}$ & $\begin{array}{c}\text { Variância } \\
\text { relativa (\%) }\end{array}$ \\
\hline \multirow{3}{*}{$\begin{array}{l}\bar{O} \\
\stackrel{\varrho}{E} \\
\text { Ũ }\end{array}$} & $20 / 05 / 2011$ & 10 & 19,73 \\
\hline & $27 / 05 / 2011$ & 15 & 14,79 \\
\hline & 03/06/2011 & 10 & 21,06 \\
\hline \multirow{3}{*}{ 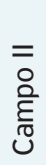 } & $20 / 05 / 2011$ & 10 & 21,59 \\
\hline & $27 / 05 / 2011$ & 10 & 23,05 \\
\hline & 03/06/2011 & 5 & 15,13 \\
\hline
\end{tabular}

curto período de tempo (Moura, 2005). Dessa forma, um plano amostral para ser considerado praticável deve requerer um tempo inferior a uma hora (Moura et al., 2003).

\section{CONCLUSÃO}

A cigarrinha (E. kraemeri) no feijão-de-corda apresenta o modelo de distribuição agregada. A distribuição binomial negativa é o modelo mais adequado para representar a distribuição da $E$. kraemeri em áreas de produção de $V$. unguiculata cultivar Vita 7. Para aplicação em programas de manejo integrado de pragas, 30 é o número de amostras adequado para a estimativa da população de E. kraemeri em campos de V. unguiculata cultivar Vita 7.

\section{AGRADECIMENTOS}

Os autores agradecem à Fundação Cearense de Apoio ao Desenvolvimento Científico e Tecnológico (Funcap), pelo apoio financeiro para a realização desta pesquisa. 


\section{REFERÊNCIAS}

BARBOSA, J.C. A amostragem sequencial. In: FERNANDES O.A.; CORREIA, A.C.B.; BORTOLI, S.A. (eds.). Manejo integrado de pragas e nematóides. Jaboticabal: FUNEP, 1992. p.205-211.

CESCONETTO, A.O.; FAVERO, S.; OLIVEIRA, A.K.M.; SOUZA, C.C. Distribuição espacial do dano da lagarta do cartucho do milho Sposoptera frugiperda (J.E. Smith, 1797), em Sidrolândia, Mato Grosso do Sul. Ensaios e Ciência, v.9, n.2, p.305-314, 2005.

ELLIOTT, N.C.; KIECKHEFER, R.W.; WALGENBACH, D.D. Binomial sequential sampling methods for cereal aphids in small grains. Journal of Economic Entomology, v.83, n.4, p.1381-1387, 1990.

FERNANDES, M.G.; BUSOLI, A.C.; BARBOSA, J.C. Distribuição espacial de Alabama argilacea (Hübner) (Lepidoptera:Noctuidae) em algodoeiro. Neotropical Entomology, v.32, n. 1, p.107-115, 2003.

GALLO, D.; NAKANO, O.; SILVEIRA NETO, S.; CARVALHO, R.P.L.; BAPTISTA, G.C.; BERTI FILHO, E.; PARRA, J.R.P.; ZUCCHI, R.A.; ALVES, S.B.; VENDRAMIM, J.D.; MARCHINI, L.C.; LOPES J.R.S.; OMOTO, C. Entomologia agrícola. Piracicaba: FEALQ, 2002. 920 p.

GILES, K.L.; ROYER, T.A.; ELLIOTT, N.C.; KINDLER, S.D Development and validation of a binomial sequential sampling plan for the greenbug (Homoptera: Aphididae) infesting winter wheat in the southern plains. Journal of Economic Entomology, v.93, n.5, p.1522-1530, 2000.

HERRERA, J.M. Primera experiencia a nivel mundial del Manejo Integrado de Plagas: el caso del algodonero en el Perú. Revista Peruana de Entomologia, v.46, n. 1, p.1-8, 2010.

KOGAN, M.; HERZOG, D.C. Sequential sampling. In: KOGAN, M.; HERZOG, D.C. (eds.). Sampling methods in soybean entomology. New York: Springer Verlag, 1980. 587p.

MARUYAMA, W.I.; BARBOSA, J.C.; TOSCANO, L.C. Distribuição espacial de Oncometopia facialis (Signoret) (Hemiptera: Cicadellidae) em pomar cítrico. Neotropical Entomology, v.35, n. 1, p.93-100, 2006.

MELO, E.P.; FERNANDES, M.G.; DEGRANDE, P.E.; CESSA, R.M.A.; SALOMÃO, J.L.; NOGUEIRA, R.F. Distribuição espacial de plantas infestadas por Spodoptera frugiperda (J.E. Smith) (Lepidoptera: Noctuidae) na cultura do milho. Neotropical Entomology, v.35, n.5, p.689-697, 2006.

MOURA, M.F. Danos, sistema de tomada de decisão de controle e distribuição espacial de Empoasca kraemeri na cultura do feijoeiro. 2005. 97f. Tese (Doutorado) - Universidade Federal de Viçosa, Viçosa, 2005.
MOURA, M.F.; PICANÇO, M.C.; GUEDES, R.N.C.; BARROS, E.C.; CHEDIAK, M.; FIDELIS, E. Conventional sampling plan for the green leafhopper Empoasca kraemeri in common beans. Journal of Applied Entomology, v. 13 1, n.3, p.215-220, 2007.

MOURA, M.F.; PICANÇO, M.C.; SILVA, E.M.; GUEDES, R.N.C. PEREIRA, J.L. Plano de amostragem do biótipo B de Bemisia tabaci na cultura do pepino. Pesquisa Agropecuária Brasileira, v.38, n. 12, p.1357-1363, 2003.

MYERS, J.H. Selecting a measure of dispersion. Environmental Entomology, v.7, p.619-621, 1978

NASERI, B.; FATHIPOUR, Y.; TALEBI, A.A. Population density and spatial distribution pattern of Empoasca decipiens (Hemiptera: Cicadellidae) on different bean species. Journal of Agricultural Science and Technology, v.11, p.239-248, 2009.

PEDIGO, L.P.; BUNTIN, G.D. Handbook of sampling methods for arthropods in agriculture. Florida: CRC Press, 1994. 714p.

PEDIGO, L.P.; RICE, M.E. Entomology and pest management. 6 ed. New Jersey: Upper Saddle River, 2009. 784p.

PEREIRA, J.L.L.; OLIVEIRA, J.V.; BARROS, R.; GONDIN JUNIOR, M.G.C.; SOUZA, A.C.F.; BARBOSA, F.T. Controle químico da cigarrinha verde Empoasca kraemeri Ross \& Moore (Homoptera: Cicadellidae) em caupi. Anais da Sociedade Entomológica do Brasil, v.22, p.497-503, 1993.

RAHMANI, H.; FATHIPOUR, Y.; KAMALI, K. Spatial distribution and seasonal activity of Panonychus ulmi (Acari: Tetranychidae) and its predator Zetzelia mali (Acari: Stigmaeidae) in apple orchards of Zanjan, Iran. Journal of Agricultural Science and Technology, v.12, n.2, p.155-165, 2010.

RODRIGUES, T.R.; FERNANDES, M.G.; SANTOS, H.R. Distribuição espacial de Aphis gossypii (Glover) (Hemiptera, Aphididae) e Bemisia tabaci (Gennadius) biótipo B (Hemiptera, Aleyrodidae) em algodoeiro Bt e não-Bt. Revista Brasileira de Entomologia, v.54, n. 1, p.136-143, 2010.

SEDARATIAN, A.; FATHIPOUR, Y.; TALEBI, A.A.; FARAHANI, S. Population density and spatial distribution pattern of Thrips tabaci (Thysanoptera: Thripidae) on different soybean varieties. Journal of Agricultural Science and Technology, v.12, p.275288, 2010.

SOUTHWOOD, T.R.E. Ecological methods: with particular reference to the study of insect populations. London: Methuen \& Co LTD., 1966. $391 \mathrm{p}$.

SOUTHWOOD, T.R.E.; HENDERSON, P.A. Ecological methods. 3 ed. Oxford: Blackwell Science, 2000. 575p. 